\title{
Henoch-Schönlein purpura in a pediatric patient with lupus
}

Peng Hu${ }^{1}$, Bao Yu Huang², Dong Dong Zhang², Guang Mei Jiang², Si Yan Liu², Xun Xia², Jie Cai ${ }^{2}$

${ }^{1}$ First Affiliated Hospital of Anhui Medical University, Hefei, China
${ }^{2}$ Department of Pediatrics, First Affiliated Hospital of Anhui Medical University, Hefei,
China

Submitted: 6 June 2015

Accepted: 5 July 2015

Arch Med Sci 2017; 13, 3: 689-690

DOI: https://doi.org/10.5114/aoms.2017.67288

Copyright $\odot 2017$ Termedia \& Banach

Systemic lupus erythematosus (SLE) is a chronic autoimmune connective tissue disease (CTD). It can involve multiple systems and lead to significant mortality. Of all cases, $10 \%$ to $20 \%$ occur in the first two decades of life, with an incidence of $0.3-0.9$ per 100,000 children-years and a prevalence of 3.3-8.8 per 100,000 children [1]. Children suffering from SLE have a more aggressive clinical course when compared with their adult counterparts [2]. In addition, it has been reported that approximately $6 \%$ to $12 \%$ juvenile-onset SLE patients may develop other concomitant CTDs within a few months, such as juvenile idiopathic arthritis, dermatomyositis, polymyositis, scleroderma and Crohn's disease [3]. In the present report, we encountered a pediatric patient who suffered from Henoch-Schönlein purpura (HSP) at 3 months after diagnosis of SLE.

A 9-year-old girl was first referred to our department in October 2014 for fever, facial butterfly erythema and polyarthralgia. The subsequent autoimmune and serologic workup came back positive for antinuclear antibody (ANA) at a titer of $1: 3200$, positive anti-double-stranded DNA (anti-dsDNA), anti-Smith antibody (anti-SM), and low complements C3 $(0.21 \mathrm{~g} / \mathrm{l})$ and $\mathrm{C} 4(0.01 \mathrm{~g} / \mathrm{l})$. Her initial blood counts were as follows: white blood cells $2.54 \times 10^{9} /$, red blood cells $3.27 \times 10^{12} /$, hemoglobin $87 \mathrm{~g} / \mathrm{l}$, and platelets $112 \times 10^{9} / \mathrm{l}$. Other blood chemistry findings were erythrocyte sedimentation rate (ESR) $34 \mathrm{~mm} / \mathrm{h}$, C-reactive protein (CRP) $12 \mathrm{mg} / \mathrm{l}$, and serum ferritin $559.90 \mu \mathrm{g} / \mathrm{l}$. Urine analysis and renal function were normal. Based on the above clinical and laboratory findings, she was diagnosed with active SLE and treated with a course of pulse methylprednisolone $15 \mathrm{mg} / \mathrm{kg} /$ day for 3 days and subsequently converted to high-dose oral prednisone $(2 \mathrm{mg} / \mathrm{kg} /$ day). After 7 days of hospitalization, the fever, facial butterfly erythema and polyarthralgia gradually resolved. Three months later, the patient presented to our department again with complaints of intermittent abdominal pain, hemafecia, and purpuric rash on her lower limbs (Figure 1). Laboratory analyses revealed leucocytosis $\left(19.18 \times 10^{9} / \mathrm{l}\right)$, thrombocytosis $\left(399 \times 10^{9} / \mathrm{l}\right)$ and an increased plasma level of immunoglobulin A (IgA, $4.24 \mathrm{~g} / \mathrm{l})$. The former high-titer ANA, positive anti-dsDNA and anti-SM, and hypocomplementemia were still existent. On these grounds, our patient was diagnosed as having SLE coexisting with HSP. After receiving the second course of pulse methylprednisolone therapy, her complaints regressed.

The HSP is an IgA-mediated autoimmune vasculitis, characterized by non-thrombocytopenic purpura, abdominal pain, arthritis and renal

\author{
Corresponding author: \\ Peng Hu PhD \\ First Affiliated \\ Hospital of Anhui \\ Medical University \\ No. 218 Jixi Road \\ 230022 Hefei, China \\ Phone: +86055162922058 \\ E-mail: hupeng28@aliyun.com
}




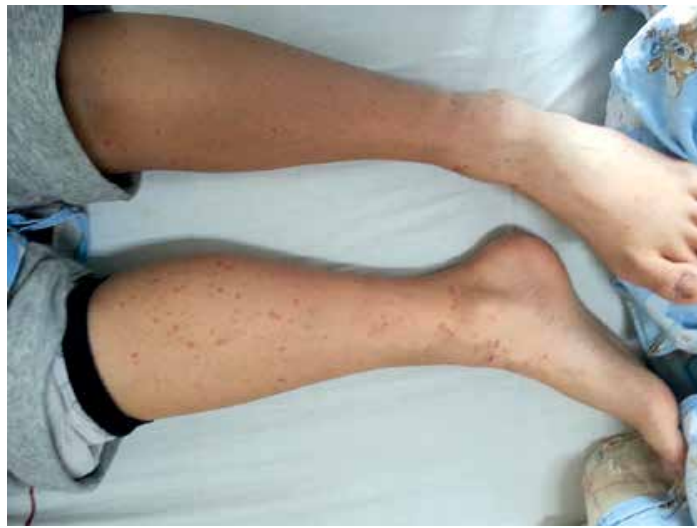

Figure 1. Palpable purpura symmetrically distributed in the lower limbs

involvement. As there is no definitive test for the diagnosis of HSP, the 1990 American College of Rheumatology criteria for HSP are still the most commonly used criteria for diagnosis [4]. In this report, our patient fulfilled three criteria (non-thrombocytopenic purpura, young age and bowel angina); thus a definite diagnosis of HSP can be made. The etiology of HSP remains unclear, and a number of potential triggers including infective agents, vaccines, drugs and insect bites have been proposed [5]. However, to date, few reports mention that HSP can be secondary to or coexisting with SLE. In 2011, Al-Attrach et al. first reported a 12-year-old girl who presented with features of HSP and was later diagnosed to have SLE [6].

In this report, we have presented an unusual child who suffered from HSP at 3 months after diagnosis of SLE. For our patient, the above two diseases shared several commonalities as well as dissimilarities. First, both SLE and HSP can cause multi-system involvement. In this case, SLE affected the skin, joints and blood, whereas HSP harmed the skin and gastrointestinal tract. Second, a characteristic rash is the hallmark of SLE and HSP. The rash of SLE is generally described as facial butterfly erythema, which often extends over the nasal bridge, affects the chin and ears, but spares the nasolabial folds [1]. The typical rash of HSP is palpable purpura and often symmetrically distributes over the extensor surfaces of the lower limbs and buttocks [4]. Third, the hemogram is usually abnormal in SLE and HSP. Leukopenia, anemia and thrombopenia are common in SLE; in contrast, the majority of patients with HSP exhibit leucocytosis and thrombocytosis [7]. However, it is worth noting that corticosteroid therapy may contribute to the elevated white blood cell and platelet count in our patients. Last, but most important, there is some evidence of associated autoimmune disorder in patients with SLE or HSP. According to the study conducted by Swart and Wulffraat, ANA and anti-dsDNA were positive in $20 \%$ and $98 \%$ patients with mixed CTD, respectively [3]. Furthermore, IgA-containing immune complexes were found in the sera of $67 \%$ of patients with HSP and $70 \%$ of patients with SLE [8].

To date, corticosteroids administered alone or combined with cytotoxic agents remain the firstline treatment for suppressing ongoing systemic inflammation in both SLE and HSP $[5,9]$. In this report, the clinical outcome of our patient was satisfactory; her complaints regressed after two courses of pulse methylprednisolone therapy with an interval of 3 months. Fortunately, renal injury and dysfunction of other organs did not occur. Juvenile-onset SLE follows a more severe disease course than adult-onset SLE, and more than $90 \%$ of patients will develop nephritis within the first 2 years after diagnosis [1]. Although HSP is usually considered as a benign disease, still $20-35 \%$ of patients progress to chronic kidney disease [10]. Therefore, the urinary analysis and renal function of our patient should be more closely monitored.

\section{Conflict of interest}

The authors declare no conflict of interest.

\section{References}

1. Levy DM, Kamphuis S. Systemic lupus erythematosus in children and adolescents. Pediatr Clin North Am 2012; 59: 345-64.

2. Lee PP, Lee TL, Ho MH, Wong WH, Lau YL. Recurrent major infections in juvenile-onset systemic lupus erythematosus-a close link with long-term disease damage. Rheumatology (Oxford) 2007; 46: 1290-6.

3. Swart JF, Wulffraat NM. Diagnostic workup for mixed connective tissue disease in childhood. Isr Med Assoc J 2008; 10: 650-2.

4. Mills JA, Michel BA, Bloch DA, et al. The American College of Rheumatology 1990 criteria for the classification of Henoch-Schönlein purpura. Arthritis Rheum 1990; 33: 1114-21.

5. Hu P, Guan Y, Lu L. Henoch-Schönlein purpura triggered by Mycoplasma pneumoniae in a female infant. Kaohsiung J Med Sci 2015; 31: 163-4.

6. Al-Attrach I, Al-Shibli A, Al-Riyami L, Al-Salam S. Systemic lupus erythematosus with severe nephritis that mimicked Henoch-Schoenlein purpura. Arab J Nephrol Transplant 2011; 4: 159-61.

7. Kraft DM, Mckee D, Scott C. Henoch-Schönlein purpura: a review. Am Fam Physician 1998; 58: 405-8, 411.

8. Hall RP, Lawley TJ, Heck JA, Katz SI. IgA-containing circulating immune complexes in dermatitis herpetiformis, Henoch-Schönlein purpura, systemic lupus erythematosus and other diseases. Clin Exp Immunol 1980; 40: 431-7.

9. Hu P, Lu L, Hu B, Fei WJ. Cyclophosphamide-induced hypertensive encephalopathy in a young girl with lupus. J Clin Hypertens (Greenwich) 2012; 14: 271-2.

10. Schärer K, Krmar R, Querfeld U, Ruder H, Waldherr R, Schaefer F. Clinical outcome of Schönlein-Henoch purpura nephritis in children. Pediatr Nephrol 1999; 13: 816-23. 